\title{
SCHUR INDICES AND SUMS OF SQUARES
}

\author{
BURTON FEIN 1
}

ABSTRACT. Let $G$ be a finite group of exponent $n, F$ a field of characteristic zero, $\epsilon$ a primitive $n$th root of unity, and suppose that the Sylow 2-subgroup of the Galois group of $F(\epsilon)$ over $F$ is cyclic. Let $\boldsymbol{X}$ be an absolutely irreducible character of $G$. Strengthening a recent result of Goldschmidt and Isaacs, it is shown that if -1 is a sum of two squares in $F$, then the Schur index of $\chi$ over $F$ is odd.

In [6], Goldschmidt and Isaacs proved the following striking generalization of the splitting field theorems of Fong, Roquette, Solomon, and Yamada:

Theorem 1 (Goldschmidt-Isaacs). Let $G$ be a finite group of exponent $n$ and let $F$ be a field of characteristic zero. Suppose for some prime $p$ that a Sylow p-subgroup $P$ of the Galois group $\operatorname{Gal}(F(\sqrt[n]{1)} / F)$ is cyclic. If 2||$P \mid$, assume also that $\sqrt{-1} \in F$. Then the Schur index over $F$ of every absolutely irreducible character of $G$ is relatively prime to $p$.

In their paper, Goldschmidt and Isaacs conjecture that the hypothesis that $\sqrt{-1} \in F$ if 2||$P \mid$ can be replaced by the weaker requirement that -1 is a sum of two squares in $F$ if 2||$P \mid$. We prove their conjecture in this note.

We denote the set of absolutely irreducible characters of $G$ by $\operatorname{Irr}(G)$. For $\chi \in \operatorname{Irr}(G)$, we denote the Schur index of $\chi$ over $F$ by $m_{F}(\chi) . \epsilon_{n}$ will denote a primitive $n$th root of unity. If $A$ and $B$ are finite dimensional central simple $F$-algebras, we write $A \sim B$ if $A$ and $B$ are similar in the Brauer group of $F . A^{r}$ will denote $A \otimes A \otimes \ldots \otimes A, r$ times.

Theorem 2. Let $F$ be a field of characteristic zero such that - 1 is a sum of two squares in $F$ and let $G$ be a finite group of exponent $n$. Assume that the Sylow 2-subgroup of $\operatorname{Gal}\left(F\left(\epsilon_{n}\right) / F\right)$ is cyclic and let $\chi \in \operatorname{Irr}(G)$. Then $m_{F}(\chi)$ is odd.

Received by the editors December 10, 1973 and, in revised form, May 24, 1974. AMS (MOS) subject classifications (1970). Primary 20C15. Key words and phrases. Schur index, division algebra. 1 This work was supported in part by NSF Grant GP-29068. 
Proof. Since $m_{F}(\chi)=m_{F(X)}(\chi)$, we may assume that $F(\chi)=F$. In view of Theorem 1 , we may assume that $\sqrt{-1} \notin F$. Since there is nothing to prove if $m_{F}(\chi)$ is odd, we assume that $2 \mid m_{F}(\chi)$. Let $Q$ denote the rational field and let $E=Q\left(\epsilon_{n}\right) \cap F$. Let $L$ be the subfield of $Q\left(\epsilon_{n}\right)$ such that $L \supset E,[L: E]$ is odd, and $\left[Q\left(\epsilon_{n}\right): L\right]$ is a power of 2. Since $Q\left(\epsilon_{n}\right)$ is a splitting field for $\chi, m_{L}(\chi)$ is a power of 2 . If $m_{L}(\chi)=1$, then $m_{L F}(\chi)=$ 1 which would imply that $m_{F}(\chi)$ is odd. If $4 \mid m_{L}(\chi)$, then $\sqrt{-1} \in L$ by the Benard-Schacher theorem [2, Theorem 1]. But then $\sqrt{-T} \in L F$ and so, since $[L F: L]=[L: F]$ is odd, we would have $\sqrt{-1} \in F$, contrary to our assumption. We conclude that $m_{L}(X)=2$.

By the Brauer-Witt theorem $[10, \S 2]$, [9] there is a hyperelementary subgroup $H$ of $G$ and $\zeta \in \operatorname{Irr}(H)$ with the following properties:

(1) there is a normal subgroup $N$ of $H$ and a linear character $\psi$ of $N$ such that $\zeta=\psi^{H}$;

(2) $H / N \cong \operatorname{Gal}(L(\psi) / L)$

(3) $L(\zeta)=L$;

(4) $m_{L}(\zeta)=2$;

(5) the simple component $A$ of the group algebra of $H$ over $L$ corresponding to $\zeta$ is isomorphic to the cyclic algebra $\left(L(\psi) / L, \sigma, \epsilon_{r}\right)$ where $\langle\sigma\rangle=\operatorname{Gal}(L(\psi) / L)$ and $\epsilon_{r} \in L$; and

(6) the simple component $B$ of the group algebra of $G$ over $L$ corresponding to $\chi$ is similar to $A$.

Since $m_{L}(\zeta)=2, A$ has index 2. Since $L$ is an algebraic number field, $A$ has exponent 2 in the Brauer group of $L\left[1\right.$, Chapter 9]. Since $A^{r} \sim$ $\left(L(\psi) / L, \sigma, \epsilon_{r}^{r}\right) \sim L$, we see that $r$ is even. Since $\sqrt{-1} \notin E$. and $[L: E]$ is odd, $\sqrt{-1} \notin L$ so $r=2 s$ where $s$ is odd. We have $A \sim(L(\psi) / L, \sigma,-1) \bigotimes_{L}$ $\left(L(\psi) / L, \sigma, \epsilon_{s}\right)$. Since $A^{2} \sim L \sim\left(L(\psi) / L, \sigma, \epsilon_{s}\right)^{2}$ and $\left(L(\psi) / L, \sigma, \epsilon_{s}\right)^{s} \sim L$, $\left(L(\psi) / L, \sigma, \epsilon_{s}\right) \sim L$. Thus $A \sim(L(\psi) / L, \sigma,-1)$.

By (6) the simple component of the group algebra of $G$ over $L F$ corresponding to $\chi$ is similar to $B \otimes_{L} L F$ and so $B \otimes_{L} L F \not L L F$. By (5), $A \otimes_{L} L F \not L F$. Let $D$ denote the usual quaternion algebra over $Q$, i.e. $D=(Q(\sqrt{-1}) / Q, \tau,-1)$. A field $K$ splits $D$ if and only if -1 is a sum of two squares in $K$. We will obtain a contradiction to the assumptions $A \otimes_{L} L F \not$ $L F$ and -1 is a sum of two squares in $F$ by proving that $A \sim D \otimes_{Q} L$.

Let $K$ be a finite extension of $L,[K: L] \leq 2$, in which -1 is a sum of two squares. We will prove that $K$ is a splitting field for $A$. If $K=L(\sqrt{-1})$, this follows from Theorem 1. Suppose $K \neq L(\sqrt{-1})$. By [5], $\sqrt{-1} \epsilon Q\left(\epsilon_{n}\right)$. 
Since $\operatorname{Gal}\left(Q\left(\epsilon_{n}\right) / L\right)$ is a cyclic 2-group, $K(\sqrt{-1})$ is the unique quadratic extension of $K$ in $K(\psi)$. $A \otimes_{L} K \sim(L(\psi) / L, \sigma,-1) \otimes_{L} K \sim(K(\psi) / K, \sigma$, - 1). To prove that $(K(\psi) / L, \sigma,-1) \sim K$ we must show that $-1 \epsilon$ $N_{K(\psi) / K}(K(\psi))$ where $N_{K(\psi) / K}$ denotes the norm from $K(\psi)$ to $K$. By the Hasse norm theorem, $-1 \in N_{K(\psi) / K}(K(\psi))$ if and only if - 1 is a local norm at every prime of $K$ [8, Theorem 4.5]. Since -1 is a sum of two squares in $K$, this also holds in every completion of $K$ and so all archimedean primes of $K$ are complex. Thus we need only consider finite primes of $K$. We calculate with the local norm residue symbol $(-1, K(\psi) / K)_{\pi}$ for any prime $\pi$ of $K[7$, Chapter $12, \S 2] .(-1, K(\psi) / K)_{\pi}=1$ if and only if -1 is a local norm at $\pi$ [7, Theorem 12-2-4]. We first consider extensions of 2 .

Let $\pi$ extend the rational prime 2. By $[4$, Theorem 1$],\left[K_{\pi}: Q_{2}\right]$ is even. We have

$$
\begin{aligned}
(-1, K(\psi) / K)_{\pi} & =\left(N_{K_{\pi} / Q_{2}}{ }^{(-1), Q(\psi) / Q)_{2}}\right. \\
& =\left(-1^{\left[K_{\pi}: Q_{2}\right]}, Q(\psi) / Q\right)_{2}=(1, Q(\psi) / Q)_{2}=1
\end{aligned}
$$

by [7, Proposition 12-2-5]. This -1 is a local norm at $\pi$ for $\pi$ extending 2 .

Since -1 is a unit, $(-1, K(\psi) / K)_{\pi}=1$ if $\pi$ is unramified from $K$ to $K(\psi)$ [8, Proposition 3.11]. Thus we may restrict our attention to those primes $\pi$ of $K$ where $\pi$ extends the rational odd prime $p, p \mid n$, and where $\pi$ is ramified from $K$ to $K(\psi)$. In particular, $K\left(\epsilon_{p}\right) \neq K$.

As noted previously, $K(\sqrt{-1})$ is the unique quadratic extension of $K$ in $K(\psi)$. Suppose $p \equiv 3(\bmod 4)$. Then $K\left(\epsilon_{p}\right)=K(\sqrt{-1})$ so $\pi$ is unramified from $K$ to $K\left(\epsilon_{p}\right)$. Since $K(\psi)$ is an extension of $K$ by roots of unity, $\pi$ is unramified from $K$ to $K(\psi)$. Thus we need only consider the case when $p \equiv 1(\bmod 4)$.

Since $p \equiv 1(\bmod 4), \epsilon_{4} \in Q_{p}[8$, Corollary 3.7]. By Theorem 1, $m_{K_{\pi}}(\zeta)=1$ and so $(-1, K(\psi) / K)_{\pi}=1$. This completes the proof that if -1 is a sum of two squares in $K,[K: L] \leq 2$, then $K$ splits $A$. In particular, -1 is not a sum of two squares in $L$ and so $D \otimes_{Q} L$ is a division algebra.

Let $D_{0}$ be the division algebra component of $A$. By [3, Corollary 2], $D_{0} \cong D \otimes_{Q} L$ if and only if $D_{0}$ and $D \otimes_{Q} L$ have precisely the same set of maximal subfields. A field $K$ is a maximal subfield of $D \otimes_{Q} L$ if and only if $[K: L]=2$ and -1 is a sum of two squares in $K$. As seen above, such fields split $A$ and so are maximal subfields of $D_{0^{*}}$. Conversely, let $[K: L]=2, K$ a splitting field for $A$. We must show that -1 is a sum of 
two squares in $K$. If $K=L(\sqrt{-1})$ we are done, so assume $K \neq L(\sqrt{-1})$. Then $(K(\psi) / K, \sigma,-1) \sim K$ and so -1 is a norm from $K(\psi)$ to $K$. Since $K(\psi) \supset K(\sqrt{-1}) \supset K,-1$ is a norm from $K(\sqrt{-1})$ to $K$, proving that -1 is a sum of two squares in $K$. This proves that $D_{0} \cong D \otimes_{Q} L$ and completes the proof of Theorem 2 .

\section{REFERENCES}

1. A. A. Albert, Structure of algebras, Amer. Math. Soc. Colloq. Publ., vol. 24, Amer. Math. Soc., Providence, R. I., 1939. MR 1, 99.

2. M. Benard and M. Schacher, The Schur subgroup. II, J. Algebra 22 (1972), 378-385. MR $46 \# 1890$.

3. B. Fein, Embedding rational division algebras, Proc. Amer. Math. Soc. 32 (1972), 427-429. MR 44 \#6756.

4. B. Fein, B. Gordon and J. Smith, On the representation of -1 as a sum of two squares in an algebraic number field, J. Number Theory 3 (1971), 310-315.

5. B. Fein and T. Yamada, The Schur index and the order and exponent of a finite group, J. Algebra 28 (1974), 496-498.

6. D. Goldschmidt and I. Isaacs, Schur indices in finite groups, J. Algebra 33 (1975), 191-199.

7. L. Goldstein, Analytic number theory, Prentice-Hall, Englewood Cliffs, N. J., 1971.

8. G. Janusz, Algebraic number fields, Academic Press, New York, 1973.

9. E. Witt, Die algebraische Struktur des Gruppenringes einer endlichen Gruppe über einem Zahlkörper, J. Reine Angew. Math. 190 (1952), 231-245. MR 14, 845.

10. T. Yamada, Characterization of the simple components of the group algebras over the p-adic number field, J. Math. Soc. Japan 23 (1971), 295-310. MR 43 \#4933.

DEPARTMENT OF MATHEMATICS, OREGON STATE UNIVERSITY, CORVALLIS, OREGON 97330 